05,13

\title{
Линейная динамика спиновых волн в массив ЖИГ-волноводов
}

\author{
(C) А.Б. Хутиева, Е.Н. Бегинин, С.Е. Шешукова, А.В. Садовников \\ Саратовский государственный университет им. Н.Г. Чернышевского, \\ Саратов, Россия \\ E-mail: abkhutieva@gmail.com
}

Поступила в Редакцию 31 июля 2021 г.

В окончательной редакции 31 июля 2021 г.

Принята к публикации 8 августа 2021 г.

\begin{abstract}
Рассмотрены особенности спин-волнового транспорта в системе связанных магнитных микроволноводов при перпендикулярном намагничивании относительно продольной оси системы. В этом случае система обладает одноосной анизотропией, коэффициенты горизонтальной и вертикальной связи имеют разные знаки, а изочастотные поверхности имеют форму „седла“. При распространении поперечно ограниченных пучков спиновых волн тип кривизны волновых фронтов определяется направлением распространения волн относительно внешнего магнитного поля.
\end{abstract}

Ключевые слова: спиновые волны, магноника, система микроволноводов, латеральные структуры.

DOI: 10.21883/FTT.2021.12.51672.181

\section{1. Введение}

Магноны, являющиеся квантами спин-волновых возбуждений, могут использоваться в качестве носителей информационных сигналов на частотах от нескольких $\mathrm{GHz}$ до сотен $\mathrm{GHz}[1]$. Магнонные сети (MC), состоящие из связанных систем спиновых микроволноводов на основе пленок железо-иттриевого граната (ЖИГ), могут использоваться для обработки информации и в то же время обеспечивать технологическую интеграцию с существующей архитектурой CMOS [2]. Например, в работе [3] было показано, что трехмерный (3D) магнонный кристалл в форме меандра может обеспечивать распространение спиновых волн во всех пространственных измерениях.

В настоящей работе мы исследуем влияние дипольной связи магнонных микроволноводов для реализации вертикального и латерального транспорта магнонов. Для исследования динамики распространения спиновых волн (CВ) в системе ЖИГ-волноводов использовался метод связанных волн.

\section{2. Система связанных волноводов и численное моделирование}

Известно, что динамика распространения волн различной физической природы в системах, состоящих из идентичных связанных волноводов может быть описана, например, исходя из метода связанных волн [4,5] в приближении ближайших соседей. В численном моделировании спинового транспорта в системе дипольносвязанных магнитных микроволноводов используется система дифференциальных уравнений первого порядка, записанная в виде [6]:

$$
\begin{aligned}
d A_{m n} / d z=i \beta A_{m n} & +i C_{v}\left(A_{m+1, n}+A_{m-1, n}\right) \\
& +i C_{g}\left(A_{m, n+1}+A_{m, n-1}\right),
\end{aligned}
$$

где: $A_{m n}-$ амплитуда спиновый волны в волноводе распространяющейся в направлении $z$, нижние индексы - номер волновода вдоль горизонтального $(m)$ и вертикального $(n)$ направлений, соответственно; $\beta-$ волновое число спиновой волны на частоте $f$ в одиночном изолированном волноводе; $C_{v}$ и $C_{g}-$ вертикальный и горизонтальный коэффициенты связи, соответственно. Динамика спиновых волн в рассматриваемой системе определяются величинами $\beta, C_{v}, C_{g}$, которые в свою очередь зависят от ориентации статического магнитного поля относительно продольного направления $z$.

В случае ориентации внешнего магнитного поля вдоль оси $z$, в магнитных микроволноводах могут распространяться обратные объемные СВ [7] и рассматриваемая система будет подобна системе связанных оптических волноводов с положительными значениями коэффициентов связи $C_{g}$ и $C_{v}[6]$.

При ориентации статического магнитного поля в плоскости $(x y)$ система становится анизотропной. Например, при намагничивании системы вдоль оси $x$, дисперсионные характеристики плоских СВ волн, распространяющихся в плоскости $(x z)$ зависят от частоты $f$ и направления волнового вектора относительно направления магнитного поля [7]. Дисперсионные характеристики плоских $\mathrm{CB}$, распространяющихся в плоскости $(y z)$ зависят только от частоты. Т.е. система связанных магнитных микроволноводов представляет собой среду с одноосной анизотропией задаваемой направлением внешнего магнитного поля. Величины этих коэффициентов зависят от геометрии и периода решетки 


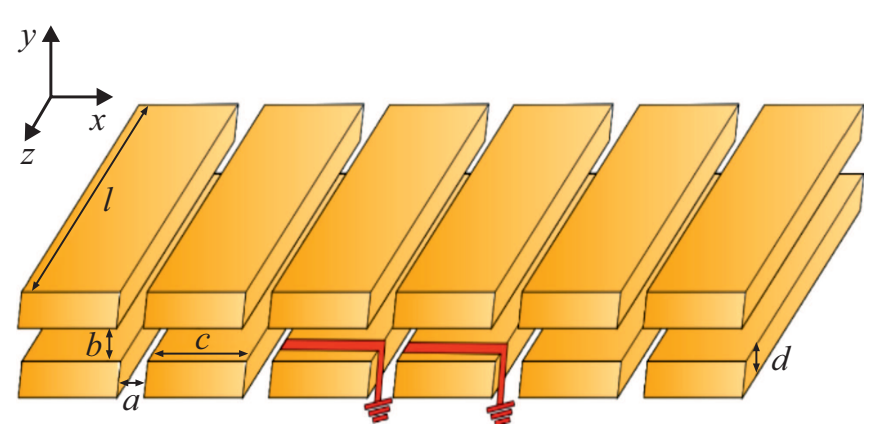

Pис. 1. Схема рассматриваемой системы связанных магнитных микроволноводов.

микроволноводов и дисперсионных характеристик $\beta(f)$ спиновых волн.

На рис. 1 схематически представлена рассматриваемая система, состоящая из идентичных магнитных ЖИГмикроволноводов шириной $c$ и толщиной $d$. Расстояния между краями смежных волноводов вдоль осей $x$ и $y$ равны $a$ и $b$, соответственно. Пространственные периоды системы по соответствующим направлениям равны $d_{x}=a+c$ и $d_{y}=b+d$. Общая длина системы волноводов $l$. Для селективной генерации спиновых волн в отдельных волноводах задавались сторонние источники динамических магнитных полей, например, в виде микрополосковых антенн. Вся система помещена во внешнее статическое магнитное поле $H$, направленное вдоль оси $x$

Рассмотрим влияние знаков коэффициентов связи $C_{v}$ и $C_{g}$ на форму изочастотных поверхностей спиновых волн в бесконечной двумерной решетке микроволноводов. Представим волновой процесс в решетке волноводов в виде плоских волн

$$
A_{m n}\left(k_{x}, k_{y}\right)=A_{0} \exp \left(i\left(k_{x} m d_{x}+k_{y} n d_{y}\right)\right) .
$$

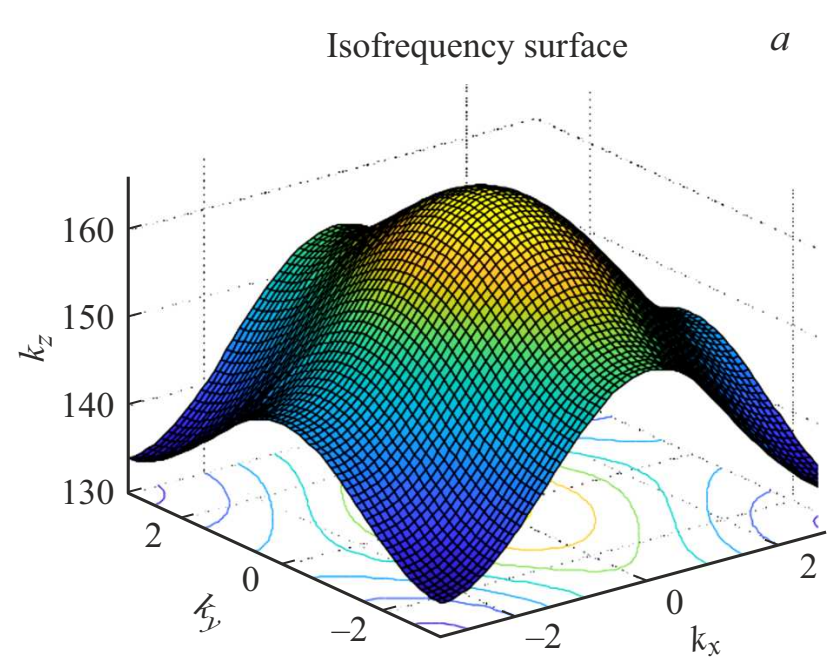

Подставляя (2) в (1) получаем дисперсионное соотношение для плоских СВ в бесконечной двумерной решетке в виде

$$
k_{z}\left(k_{x}, k_{y}\right)=\beta+2 C_{g} \cos \left(k_{x} d_{x}\right)+2 C_{v} \cos \left(k_{y} d_{y}\right) .
$$

На рис. 2 представлены результаты расчетов на фиксированной частоте $f$ зависимостей $k_{z}\left(k_{x}, k_{y}\right)$ (изочастотные поверхности) при различных знаках коэффициентов связи. В случае коэффициентов связи одного знака (рис. 2, a) изочастотная поверхность имеет форму „вала“ с максимумом волнового числа $k_{z}$ в точке с координатами $(0,0)$ и подобна изочастотным поверхностям в случае оптических волноводов. Когда коэффициенты связи имеют разные знаки (рис. 2,b) изочастотная поверхность имеет форму „седла“ с минимумом волнового числа $k_{z}$ в точке с координатами $(0,0)$. Такая форма изочастотной поверхности обусловлена видом дисперсионных характеристик поверхностных $\mathrm{CB}$, распространяющихся под различными углами к направлению статического магнитного поля [7].

Рассмотрим особенности формирования пучков спиновых волн в бесконечной двумерной решетке волноводов при возбуждении единственного волновода (точечный источник возбуждения). На рис. 3 представлены пространственные распределения амплитуд спиновых волн в двух взаимно перпендикулярных плоскостях $(x z)$ и $(y z)$ проходящих через источник возбуждения и различающимися знаками коэффициентов связи $C_{v}>0$ и $C_{g}<0$. Как видно из представленных результатов наблюдается дифракционное расплывание пучка спиновых волн за счет связи между смежными волноводами. При этом формы волновых фронтов в двух взаимно перпендикулярных плоскостях различаются: в плоскости $(x z)$ по отношению к источнику фронт вогнутый, а в плоскости $(y z)$ - выпуклый. Указанные особенности дисперсионных характери-

Рис. 2. Изочастотные поверхности спиновых волн при $\beta=150 \mathrm{~cm}^{-1}$ в бесконечной решетке волноводов: $a-C_{v}=3.0 \mathrm{~cm}^{-1}$, $C_{g}=5.0 \mathrm{~cm}^{-1} ; b-C_{v}=3.0 \mathrm{~cm}^{-1}, C_{g}=-5.0 \mathrm{~cm}^{-1}$. 


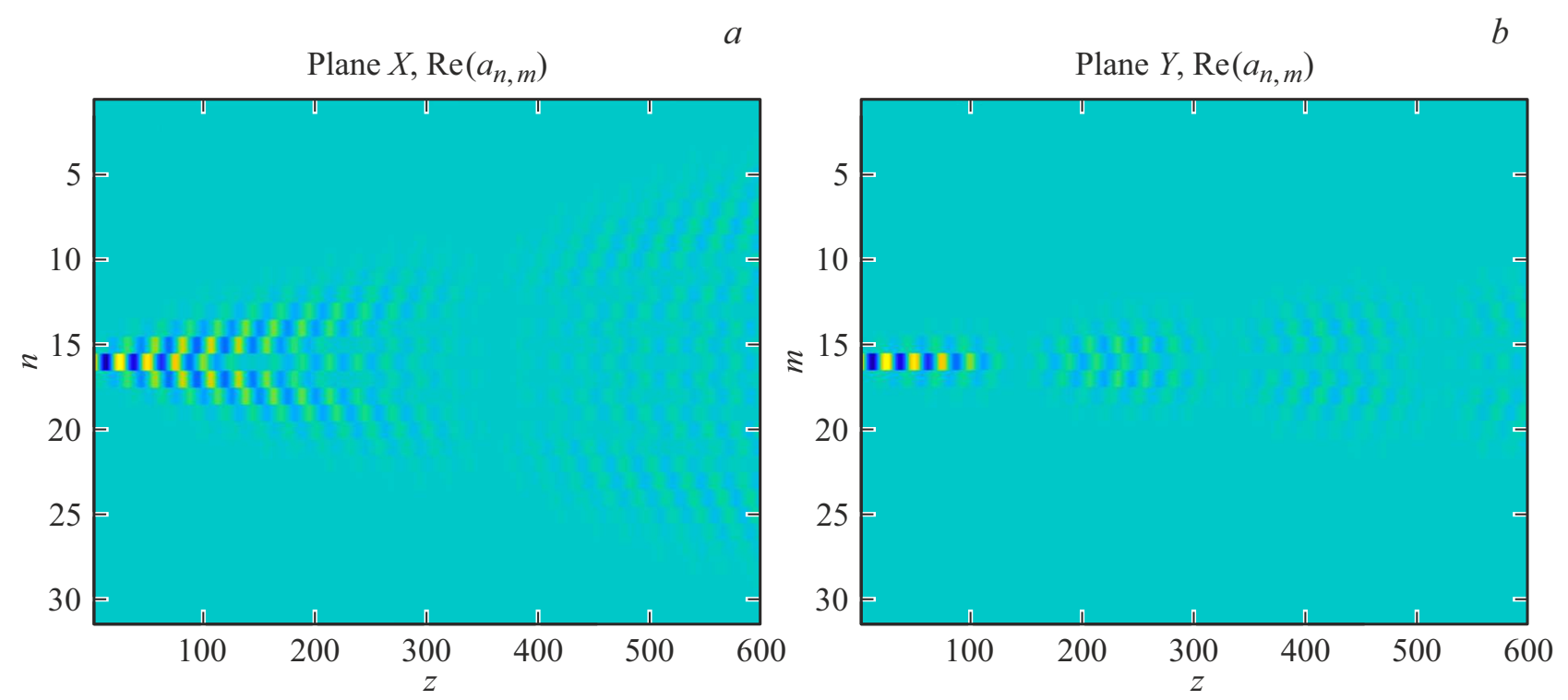

Рис. 3. Пространственные распределения амплитуд спиновых волн в двумерной решетке волноводов при $\beta=150 \mathrm{~cm}^{-1}$, $C_{v}=2.0 \mathrm{~cm}^{-1}, C_{g}=-5.0 \mathrm{~cm}^{-1}: a-$ плоскость $x z, b$ - плоскость $y z$.

стик СВ приводят к формированию сложных волновых паттернов в системах связанных магнитных микроволноводов.

\section{3. Заключение}

Таким образом, проведенные исследования линейной динамики спиновых волн в системе связанных ЖИГ волноводов показали, что при намагничивании системы перпендикулярно направлению распространения СВ она обладает одноосной анизотропией и в рамках метода связанных волн может быть описана путем введения положительных и отрицательных коэффициентов горизонтальной и вертикальной связи. При распространении поперечно ограниченных пучков спиновых волн тип кривизны волновых фронтов (выпуклый, вогнутый) определяется направлением распространения СВ относительно внешнего магнитного поля. Полученные результаты могут быть использованы для уточнения характера спин-волнового транспорта в массивах микрои наноразмерных магнитных структур.

\section{Финансирование работы}

Работа выполнена при поддержке Российского фонда фундаментальных исследований (№ 19-29-03034) и гранта Президента Российской Федерации (МК1870.2020.9).

\section{Список литературы}

[1] V.V. Kruglyak, S.O. Demokritov, D. Grundler. J. Phys. D 43, 264001 (2010).

[2] A.V. Sadovnikov, S.A. Nikitov, E.N. Beginin, A.V. Sadovnikov, E.N. Beginin, S.E. Sheshukova, Yu.P. Sharaevskii, A.I. Stognij, N.N. Novitski, V.K. Sakharov, Yu.V. Khivintsev, S.A. Nikitov. Phys. Rev. B 99, 0544242019.

[3] G. Gubbiotti, A. Sadovnikov, E. Beginin, S. Nikitov, D. Wan, A. Gupta, S. Kundu, G. Talmelli, R. Carpenter, I. Asselberghs, I.P. Radu, C. Adelmann, F. Ciubotaru. Phys. Rev. Appl. 2021.

[4] A. Yariv. IEEE J. Quantum Electron. 9, 9, 919 (1973).

[5] W.-P. Huang. J. Opt. Soc. Am. 11, 3, 963 (1964).

[6] F. Lederer, G.I. Stegeman, D.N. Christodoulides, G.A. Assanto, M. Segev, Y. Silberberg. Phys. Rep. 463, 1-3, 1 (2008).

[7] D. Stancil, A. Prabhakar. Spin Waves: Theory and Applications. Springer, N.Y. (2009). 346 p.

Редактор Д.В. Жуманов

\section{Конфликт интересов}

Авторы заявляют, что у них нет конфликта интересов. 